\title{
Measuring Density Profiles of Electrons and Heavy Particles in a Stable Axially Blown Arc
}

\author{
J. Carstensen, ${ }^{*}$ P. Stoller, B. Galletti, C. B. Doiron, and A. Sokolov \\ ABB Switzerland Ltd., Corporate Research, Segelhofstrasse 1K, 5405 Baden-Dättwil, Switzerland \\ (Received 9 December 2016; revised manuscript received 8 May 2017; published 2 August 2017) \\ Two-color spatial carrier wave interferometry employing pulsed 532- and 671-nm lasers is used to \\ measure the electron-density and heavy-particle-density profiles in the stagnation point of a stable, axially \\ blown arc in argon for currents of 50 to $200 \mathrm{~A}$ and stagnation point pressures of 0.2 to $16 \mathrm{bar}$. This technique \\ takes advantage of the fact that the free-electron contribution to the refractive index depends strongly on the \\ wavelength, while that of the heavy particles does not. The high spatial resolution achieved allows the hot \\ core of the arc to be readily distinguished from the surrounding boundary layer. A custom-built test device is \\ used to ensure flow conditions that lead to a stable, axisymmetric arc; this permits the reconstruction of the \\ density and temperature profiles using a single projection (interferometric image) of the refractive-index \\ distribution through the arc (at two wavelengths). The arc radius determined from the heavy-particle density \\ decreases with increasing stagnation pressure and increases with the current. These measurements are in \\ good agreement with a simple axially blown arc model taking into account Ohmic heating, radiation losses, \\ and enthalpy flow for core temperatures of approximately $16500 \mathrm{~K}$. The measured electron density at the \\ center of the arc agrees well with a prediction based on local thermodynamic equilibrium.
}

DOI: 10.1103/PhysRevApplied.8.024002

\section{INTRODUCTION}

Electrical arcs have been the subject of extensive scientific research since Franklin's [1] work in this field in the 18th century. Nevertheless, the complex physics of the arc has not been completely understood and cannot be modeled in a simple way. The transition from the hot (with temperatures of the order of $10000 \mathrm{~K}$ ) ionized core of the arc to the surrounding gas, which is at ambient temperature, is characterized by strong gradients and is one of the most important aspects of arc physics. One of the key processes that defines the density, temperature, and conductivity profiles of an arc is radiation transport, a topic that has been the subject of numerous theoretical and computational studies including Refs. [2-6] to name only a few papers that have focused on finding a simplified, efficient modeling approach. However, there are only limited experimental studies of the arc profile [7-12], particularly, the high-resolution, spatially resolved measurements needed to validate numerical models of the arc. In a recent survey of the field of thermal plasma modeling, Gleizes [13] emphasizes the importance of experimental validation and the limited number of benchmarks available.

Of particular benefit for validating simulations are results obtained in stable, cylindrically symmetric arcs that are not

\footnotetext{
* Corresponding author. jan.carstensen@ch.abb.com

Published by the American Physical Society under the terms of the Creative Commons Attribution 4.0 International license. Further distribution of this work must maintain attribution to the author(s) and the published article's title, journal citation, and DOI.
}

influenced by material eroded from the arcing contacts. Such an arc can be achieved in a configuration that stabilizes the arc in an axially blown, bidirectional flow of uncontaminated gas, a configuration reminiscent of gas circuit breakers. Current interruption is an important application of arcs; it takes advantage of the rapid transition from high conductivity to an insulating gap achieved by cooling an arc. For more than half a century, gas circuit breakers have been used to interrupt nominal and shortcircuit currents in the alternating-current power transmission grid. Such circuit breakers function by first opening a pair of nominal contacts to transfer the current to a pair of arcing contacts. When the arcing contacts separate within milliseconds afterwards, an arc is drawn between them. This arc is cooled by a transonic gas flow driven by a pressure difference that is provided by a prepressurized volume or generated mechanically or by the arc itself. In most configurations, the gas flow is parallel to the axis of the arc and accelerates from a stagnation point. The acceleration of the gas to speeds at or near the speed of sound allows it to cool the arc while stabilizing it on its axis [14]. When the current approaches a zero crossing, turbulent heat conduction and convective cooling lead to the interruption of the arc channel and the flow of current [15].

Thus, spatially resolved measurements of the density, temperature, or conductivity of an axially blown arc can lead to improved understanding of fundamental processes, such as radiation transport in an arc, and it can give insights useful in the development of improved circuit breakers. Such measurements can be performed using nonintrusive optical diagnostic techniques. Optical techniques can be 
loosely divided into two categories: those that measure the index of refraction and those that measure the emission spectrum. The latter techniques provide useful information about the temperature distribution in the arc during the high-current phase $[16,17]$. In the study of current interruption, the phase just before, at, and immediately after the zero crossing determines if the current is interrupted. Thus, optical techniques that rely on emission spectroscopy have only limited applicability. Refractive-index-based techniques, which rely on an external light source, can be used during the high-current phase (provided the laser outshines the arc with the help of appropriate narrow-band filters) and the current-zero phase. Recently, speckle imaging was applied to imaging axially blown arcs in test devices representative of circuit breakers [12]. Inada et al. [11] used a related technique, two-color Shack-Hartmann interferometry, to measure the spatially resolved electron density in air and in $\mathrm{SF}_{6}$ arcs, an approach applied earlier by Ref. [18] for measurements on the axis of a wallstabilized arc burning in $\mathrm{SF}_{6}$ and $\mathrm{Ar}_{-} \mathrm{SF}_{6}$ mixtures. The approach of Ref. [11] is particularly interesting for the study of circuit-breaker arcs near current zero because it has the potential to provide information about the heavyparticle density (and, given information about the pressure distribution, the temperature) and the electron density in the phase leading to current zero simultaneously. Thomson scattering, which falls into neither of the two categories above, can also be used to measure the electron density and temperature in an arc [19]. It is difficult, however, to obtain spatially resolved data with this technique [20].

One of the key disadvantages of the test devices used in recent studies $[11,20]$ is the instability of the arc. To some extent, this instability can be overcome by choosing a sufficiently short measurement time scale (i.e., a nanosecond laser pulse). In this case, instability and movement of the arc on a short time scale does not lead to smearing or averaging of features. However, the asymmetry of the arc remains. This asymmetry prevents an accurate reconstruction of, for example, the density distribution, since a single two-dimensional image (projection) can be used only to reconstruct a three-dimensional projection if it is axially symmetric (or has some other known symmetry). In Ref. [12], many images were acquired and those which were most cylindrically symmetric were selected. Stoffels et al. [21] attempted to overcome this problem by developing a tomographic technique with four projections that permits the reconstruction of asymmetric features. However, even with four projections, the spatial resolution was too limited to reconstruct an asymmetric arc and extension to more projections would have added greatly to the cost of the experiment. In Ref. [20], the Thomson scattering setup had to be modified with a cylindrical lens to perform measurements on an unstable arc. A different approach to address the problem of arc instability and asymmetry is to focus on stabilizing the arc. In Ref. [22], an approach for generating a stable, cylindrically symmetric axially blown arc with currents of up to $5 \mathrm{kA}$ that is subsequently ramped to zero was presented. Instead of generating a high pressure in an upstream volume that was exhausted to a low-pressure volume, axially blowing the arc in the process, the arc zone and surrounding pressure vessel were brought to a high pressure. Flow was then initiated by triggering the bursting of a diaphragm. The gas accelerated from rest, eliminating the upstream turbulence that enters the arc zone and destabilizes the arc in a device with a separate high-pressure volume as the source of the flow. We adopt the basic ideas behind that approach and design a test device that enables rapid, repeated experiments. The diaphragm is replaced by a mechanically operated plate that is opened using a circuit-breaker drive to initiate gas flow. In addition, unlike in the test device of Ref. [22], the contacts separate to ignite the arc; no ignition wire is needed.

In this work, we combine spatial carrier wave interferometry $[23,24]$ and two-color laser interferometry [25] to measure the heavy-particle and electron density of a stable, axially blown arc. We perform these measurements for upstream pressures ranging from roughly 2 to 16 bar and for quasi-direct-current (dc) currents of 50 to $200 \mathrm{~A}$, typical of the currents seen in high-voltage circuit breakers in the microseconds before a current-zero crossing. Unlike techniques such as speckle, Shack-Hartmann, and schlieren imaging, the interferometric approach measures the lineintegrated refractive index directly; there is no need to integrate the gradient of the refractive index, which is the parameter measured by the other techniques.

The test device and the optical setup used to generate a stable, axially blown arc are presented in Sec. II, followed by a brief summary of the theoretical basis for determining the heavy-particle and electron densities from measurements of the index of refraction at two wavelengths. The results of measurements in argon for different quasi-dc currents and blowing pressures are presented and discussed in the sections that follow.

\section{EXPERIMENT}

\section{A. Test device to generate a stable, axially blown arc}

To obtain a stable, cylindrically symmetric arc, a test device is constructed that ensures that the gas flow that cools the arc accelerates from rest away from a stagnation point in both directions, analogous to the test device described in Ref. [22]. The arcing zone of the test device is shown in Fig. 1. The device consists of an aluminum pressure vessel and lid and a series of metallic and polytetrafluoroethylene (PTFE) disks. The PTFE disks are equipped with quartz glass windows to provide optical access to the arc zone. The axial extent of the glass window in the stagnation region is limited to $10 \mathrm{~mm}$ and that of the other windows to $5 \mathrm{~mm}$. Limiting the thickness of the glass 


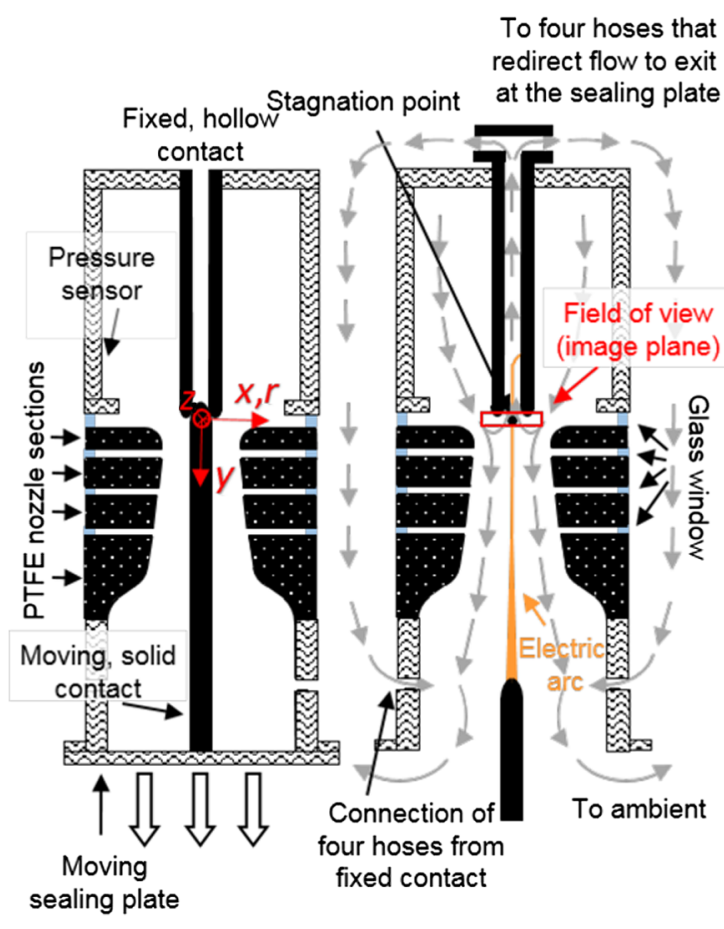

FIG. 1. Sketch of the test device in closed and open state (not true to scale). The location of the pressure sensors is indicated. The HMC 4 drive is mounted at the bottom and moves the sealing plate down. Gas that flows through the upper contact and the metal cylinder that holds it is redirected to the bottom of the test device via four hoses. The approximate field of view of the interferometer is indicated by the red rectangle. $y=0$ is at the end of the upper hollow contact and $x=0$ and $r=0$ on the axis of the test device. The laser propagates along the $z$ axis (normal to the field of view). The coordinate system shown is used throughout this paper. The image plane (or field of view) indicated in Fig. 2 is located on the axis of symmetry of the arc and experimental setup. The gray arrows indicate the direction of the gas flow through the nozzle and contact system. The stagnation point is located near the origin of the coordinate system.

windows minimizes the influence of the resulting discontinuity in the nozzle profile on the flow. The test device is closed using a pressure-tight sealing plate. The sealing plate is opened using an HMC-4 spring-hydraulic operating mechanism that moves the sealing plate down approximately $20.5 \mathrm{~cm}$ in roughly $30 \mathrm{~ms}$, initiating the flow of gas, and at the same time, pulling the moving contact away from the upper, fixed contact to draw an arc when a current flows. The gas flow through the nozzle and the upper, hollow contact cool and stabilize the arc. The gas flow through the upper, hollow contact is exhausted to the lower part of the test device using hoses. Prior to each test, the air is evacuated from the test device, and it is filled with argon from a gas cylinder using a precision pressure regulator until the specified pressure is reached (in the range of about 2 to 16 bar). The test device is equipped with four Kistler 4080A-020 0- to 20-bar pressure sensors (indicated in Fig. 1), one of which is located at the edge of the pressure vessel in the plane of the stagnation zone.

\section{B. Two-color interferometer}

Two $Q$-switched pulsed lasers, a CrystaLaser QL 532100 and a CNI AO-V-671, operating at 532 and $671 \mathrm{~nm}$ and with nominal pulse widths of 12 and $16 \mathrm{~ns}$, respectively, are combined using a beam-splitter cube. Roughly $50 \%$ of the combined beam leaving the beam-splitter cube is used as a reference beam, which is split into two beams again using a dichroic beam-splitter cube and expanded using Thorlabs 1:20 beam expanders before it is incident on the camera. The remaining $50 \%$ of the combined beam - the probe beam-is expanded using a 1:20 beam expander and passes through the window in the test device. A telecentric lens system with a magnification of 2.5 is used to image the arc zone (at the axis of the test device) onto two camera sensors (Prosilica GT 4905 and Prosilica GT 2300). A polarizing beam-splitter cube is used to split the probe beam between the two cameras. The green laser is $p$ polarized, while the red laser is $s$ polarized with respect to the beam-splitter cube to minimize the intensity loss of the probe beams within the beam-splitter cube. The 532- and 671-nm reference beams interfere with the probe beam on the Prosilica GT 2300 and Prosilica GT 4905 cameras, respectively. The angle of the reference beam with respect to the probe beam is varied to select an appropriate spatial carrier wave, as we discuss in more detail below. The intensity of the two lasers in both the reference and probe beams is adjusted using half-wave plates and polarizers so that they have roughly the same intensity. Narrowbandpass filters are used to allow the laser light to pass while filtering out light emitted by the arc outside of the bandpass range.

The two lasers are triggered to emit single pulses simultaneously, and the two cameras are triggered to record the resulting interferogram during the quasi-dc phase of the current applied to the test device (described below). For each measurement, camera images are acquired for a test with an axially blown arc and for a reference test without an axially blown arc (no current and no gas flow).

\section{Electrical circuit}

The electrical circuit used to generate the test current consists of two branches parallel to the test object as illustrated in Fig. 3(a). The first branch [left-hand side of Fig. 3(a)] is an $L-C$ circuit used to keep the arc burning for a sufficiently long time to open the test device and to establish a stationary gas flow. The second branch [righthand side of Fig. 3(a)] is an $R$ - $C$ circuit that is used as a stiff current source to reduce the arc network interaction and thereby provide a more stable arc. The following procedure is used to generate the current signal given in Fig. 3(b). In the beginning, the closing switch is open, the auxiliary breaker is closed, and the two capacitors $C_{\mathrm{HC}}$ and $C_{\mathrm{HV}}$ are charged to 4 and $50 \mathrm{kV}$, respectively. When the closing switch is triggered, a low-frequency (approximately $10 \mathrm{~Hz}$ ) oscillating current is generated that is interrupted after one 


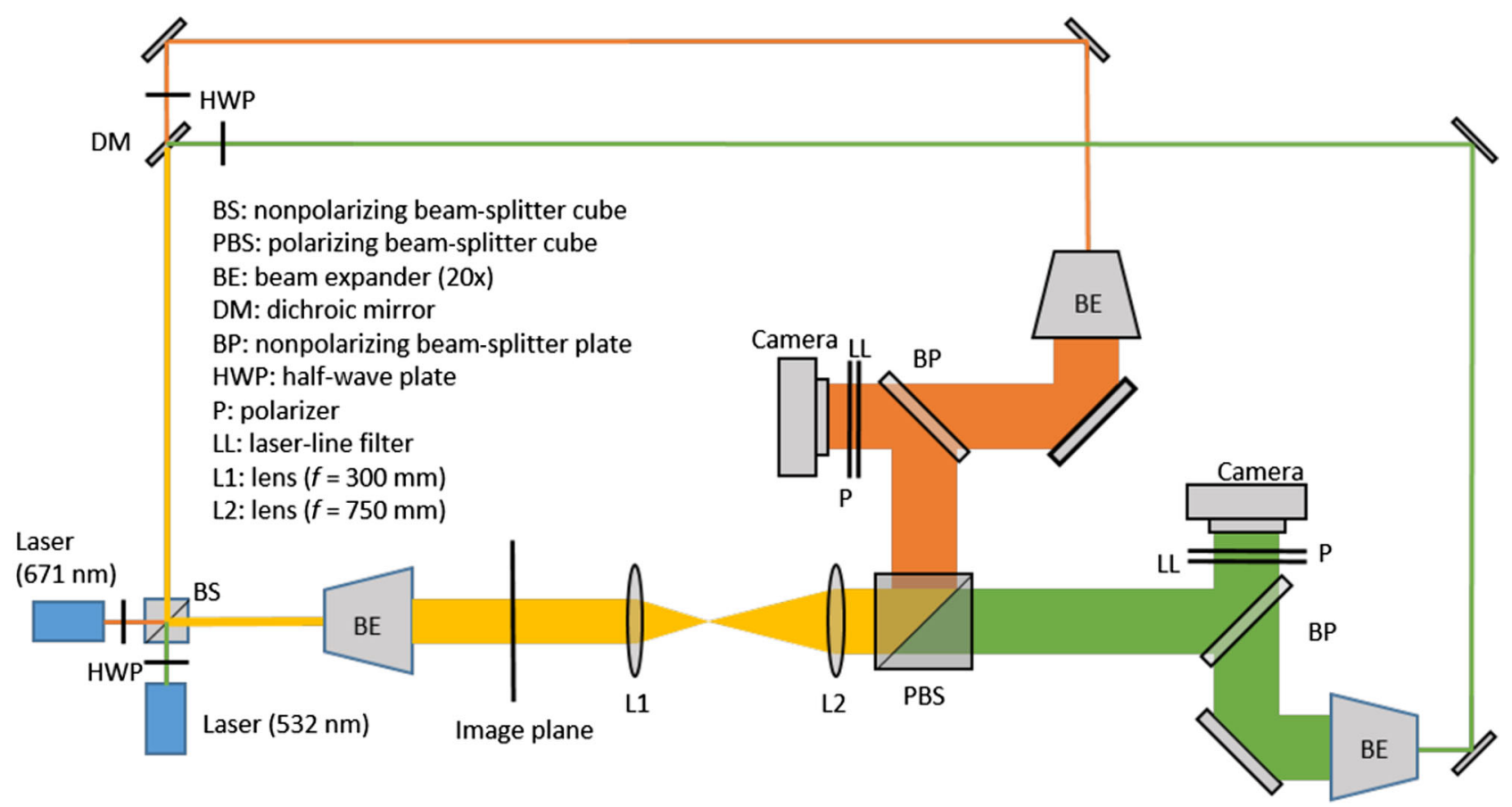

FIG. 2. Sketch of the experimental setup for two-color spatial carrier wave interferometry. (Distances not true to scale.)

half wave by the auxiliary circuit breaker. The contacts of the test device are separated, and gas flow is initiated just after the start of electric current flow, and at the end of the half wave, the fully open position is reached. Just before the end of the half wave, the spark gap is triggered, and a slowly decaying (quasistationary) current is generated. All measurements that are shown in the following are performed in this quasistationary current phase after the $L-C$ oscillation is interrupted by the auxiliary circuit breaker.
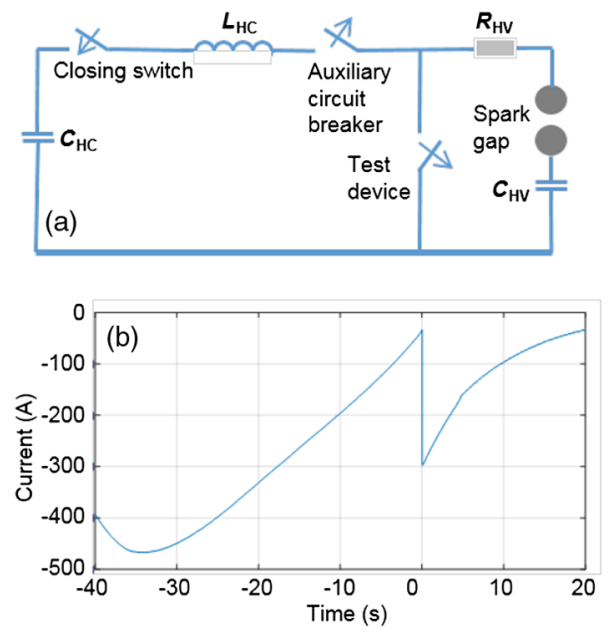

FIG. 3. (a) Sketch of the electrical circuit used in the tests. (b) Current waveform generated by the electrical circuit. The interferometry measurement is performed during the $R-C$ decay of the injected quasi-dc current at $t=9 \mathrm{~ms}$ and a current of roughly $100 \mathrm{~A}$.
Measurements at different currents are realized by measuring at different points in time of the $R-C$ decay, e.g., between 8 and $20 \mathrm{~ms}$ in Fig. 3(b).

\section{THEORY AND DATA ANALYSIS}

\section{A. Spatial carrier wave interferometry}

The probe beam that propagates through the phase object, i.e., the arc and surrounding gas, acquires an integrated phase shift $\Phi(x, y)$ with respect to the reference beam. To measure this phase shift, we introduce a spatial carrier as proposed by Takeda et al. [24]. This is achieved by adjusting the probe beam and the reference beam so that the latter is offset by a small angle from the former. This small angular offset between the probe beam and the reference beam can be expressed by the following approximations for the spatial dependence of the electric field of the probe and reference beams, respectively, in the plane (represented by $x-y$ coordinates) normal to the propagation direction of the probe beam (defined to be the $z$ axis):

$$
\begin{gathered}
E_{\text {probe }}(x, y)=E_{p}(x, y) e^{i \Phi(x, y)}, \\
E_{\text {ref }}(x, y)=E_{r} e^{i\left(k_{x}^{0} x+k_{y}^{0} y\right)},
\end{gathered}
$$

where $E_{p}(x, y)$ and $\Phi(x, y)$ are the spatial dependence of the amplitude and phase, respectively, of the probe beam electric field, $E_{r}$ is the amplitude of the reference beam electric field, and $k_{x}^{0}$ and $k_{y}^{0}$ are the wave-vector components of the reference beam electric field. The angle between the probe and the reference beam when they impinge on the camera is 
given by $\theta=a \tan \sqrt{k_{x}^{02}+k_{y}^{02}} / k_{z}$. The intensity measured by the camera is given by

$$
\begin{aligned}
I(x, y)= & I_{o}(x, y)+E_{\text {probe }}(x, y) E_{r} e^{i\left(k_{x}^{0} x+k_{y}^{0} y\right)} \\
& +E_{\text {probe }}^{*}(x, y) E_{r} e^{-i\left(k_{x}^{0} x+k_{y}^{0} y\right)},
\end{aligned}
$$

where $I_{o}$ represents the background intensity due to the components of the probe and reference beams that do not interfere. The Fourier transform of the intensity can then be expressed as

$$
\begin{aligned}
\mathscr{F}[I(x, y)]= & \tilde{I}\left(k_{x}, k_{y}\right)=\tilde{I}_{o}\left(k_{x}, k_{y}\right)+C\left(k_{x}-k_{x}^{0}, k_{y}-k_{y}^{0}\right) \\
& +C^{*}\left(k_{x}+k_{x}^{0}, k_{y}+k_{y}^{0}\right),
\end{aligned}
$$

where

$$
C=\mathscr{F}\left[\frac{1}{2} E_{\text {probe }} E_{r}\right] .
$$

The power spectrum of the example interference pattern in Fig. 4(a) is shown in Fig. 4(b). The black circle in the figure corresponds to the term $C$ in the Fourier transform. It can be seen that in Fourier space, the term that corresponds to interference between the probe and reference beam can be well separated from the rest of the interference pattern. The phase of the inverse Fourier transform of $C$ is $\Phi(x, y)$. To minimize the effect of small disturbances within the optical path on the measured phase, two interferograms are recorded per measurement: one interferogram with the phase object present and one without it present (the test device is filled with ambient air; there is no arc and no gas flow). Thus, two phase measurements are obtained, $\Phi_{1}$ and $\Phi_{2}$, and the phase shift due to the phase object (arc embedded in transonic gas flow) referenced to conditions without arc and flow is $\Delta \Phi=\Phi_{2}-\Phi_{1}$.

\section{B. Validation of the interferometry technique}

To test the accuracy of the method detailed above, we measure the phase shift of a weak lens analogous to the approach employed in Ref. [26]. To adapt the field of view of the interferometer to the size of the lens, the magnification is changed to 0.4 (instead of 2.5) for this measurement. In the quadratic approximation, the phase shift due to the lens is given by $\Delta \phi(x, y)=[(\pi) / \lambda f]\left[\left(x-x_{0}\right)^{2}+\right.$ $\left.\left(y-y_{0}\right)^{2}\right]+\phi_{0}$. The position defined by the coordinates $\left(x_{0}, y_{0}\right)$ represents the center of the lens, $\phi_{0}$ is a constant offset, and $[\pi /(\lambda f)]$ is a measure of the curvature of the phase shift. The measured phase shift (blue dots) and a parabolic surface fit are illustrated in Fig. 5(a). In addition, a cut through this parabolic surface in the $y$ direction through $y_{0}$ is shown in Fig. 5(b) for better readability. It should be noted that the measured phase shift must first be unwrapped; the algorithm presented in Ref. [27] and
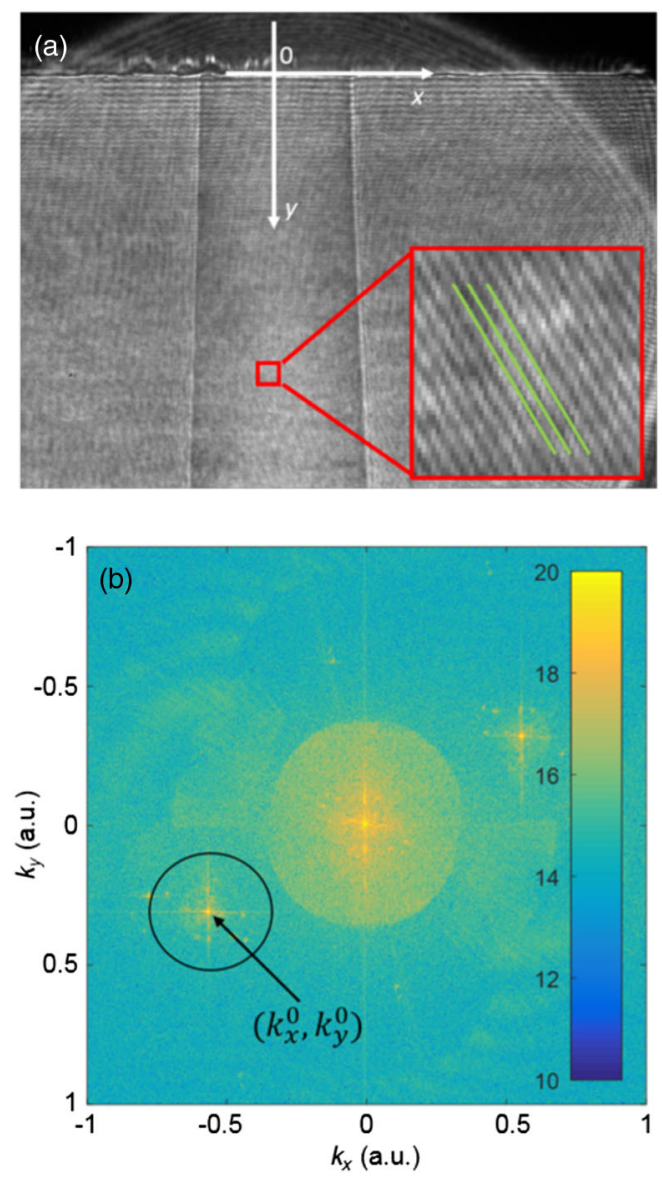

FIG. 4. (a) Example of an interference pattern for the 532-nm laser illustrating the zero-order interference between the probe and reference beams. (b) Color-coded power spectrum of the interferogram shown in (a). The color axis is logarithmic and in arbitrary units.

implemented in MATLAB in Ref. [28] is used for this purpose. The focal length $f=11.36 \mathrm{~m}$, as determined from fitting the formula for $\phi(x, y)$ above to the measured data, is in good agreement with the nominal focal length of $f=11.18 \mathrm{~m}$ (for a wavelength of $\lambda=532 \mathrm{~nm}$ ). This serves as verification of the correct implementation of the spatial carrier wave interferometry technique.

\section{Determination of the refractive index}

The index of refraction $n$ can readily be calculated from the measured phase shift and the wavelength $\lambda$ :

$$
\Delta \Phi=\int \frac{2 \pi \Delta n}{\lambda} d z=\frac{2 \pi \Delta \bar{n}}{\lambda} .
$$

Only the contribution of the axially blown arc (and surrounding gas flow) to the refractive index is of interest. The contribution from the atmospheric air through which the laser propagates is subtracted by obtaining a reference image and determining $\Delta \Phi$ as we discuss above. To keep 


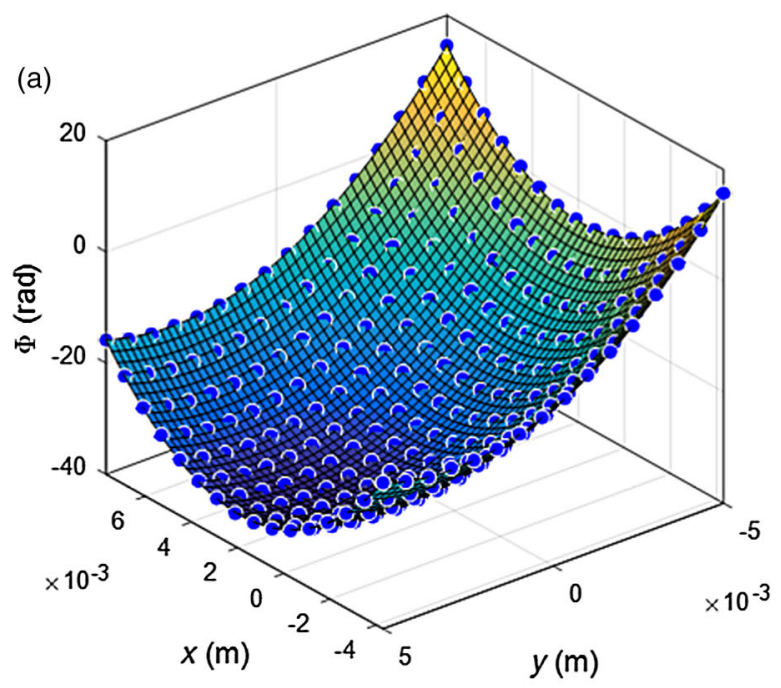

(b)

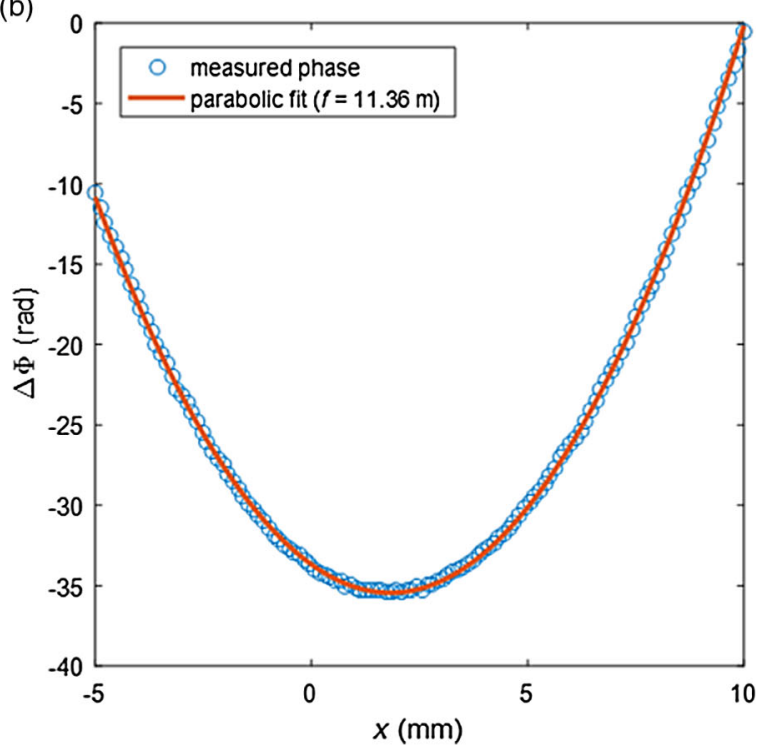

FIG. 5. (a) Unwrapped phase-shift distribution measured for a simple lens with a nominal focal length of $11.18 \mathrm{~m}$ at $532 \mathrm{~nm}$. (b) Unwrapped phase shift measured for a simple lens with a nominal focal length of $11.18 \mathrm{~m}$ at $532 \mathrm{~nm}$ along a cut through the center (blue circles). The solid red line is a parabolic fit that corresponds to a focal length of $11.36 \mathrm{~m}$.

the notation consistent, we use $\Delta n$ to represent the contribution to the line-integrated index of refraction $\Delta \bar{n}$ that stems from the axially blown arc and the surrounding gas flow.

As detailed in Ref. [12], Abel inversion can be used to calculate the spatial distribution of $\Delta n$ since the arc and gas flow imaged are cylindrically symmetric. This cylindrical symmetry is illustrated in Video 1 . The index of refraction at the two different wavelengths used, in turn, can be employed to calculate the heavy-particle and electron densities, as we detail below. However, Abel inversion can amplify the noise in the measured line-integrated index

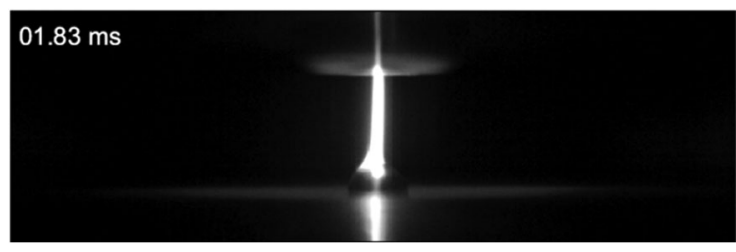

VIDEO 1. Video of the arc in the stagnation zone during and after separation of the contacts acquired using a Phantom V711 camera for a current of $10 \mathrm{~A}$ and a stagnation pressure of 5 bar in $\mathrm{CO}_{2}$.

of refraction data. Therefore, it is advantageous to calculate line-integrated quantities first - such as the line-integrated electron density - and perform the Abel inversion in a final step.

\section{Calculation of the heavy-particle and electron densities}

We consider the contribution of the heavy particles $n_{H}$ and the electrons $n_{e}$ to the index of refraction separately. We assume that the Gladstone-Dale relation [29] accurately describes the contribution of the heavy particles to the index of refraction for all temperatures up to those ( $>10000 \mathrm{~K}$ for argon) at which it becomes negligibly small,

$$
n_{H}-1=\alpha(\lambda) \rho_{H},
$$

where $\alpha$ is the Gladstone-Dale coefficient. The GladstoneDale coefficient depends only weakly on the wavelength, and in some cases, this dependence is neglected [30]. Based on the dispersion relation for argon given in Ref. [31] we use $\alpha_{g}=1.596 \times 10^{-4} \mathrm{~m}^{3} / \mathrm{kg}$ and $\alpha_{r}=1.585 \times$ $10^{-4} \mathrm{~m}^{3} / \mathrm{kg}$ for the green and the red laser, respectively. Analogous to $\Delta n$, we define $\Delta \rho_{H}=\rho_{H}-\rho_{H 0}$. The refractive index of the free electrons (neglecting pressure effects and collisions) is given by

$$
n_{e}=\sqrt{1-\frac{\omega_{p e}^{2}}{\omega^{2}}}
$$

where $\omega_{p e}=\sqrt{\left[\left(N_{e} e^{2}\right) / \epsilon_{0} m_{e}\right]}$, and $\omega$ represents the electron plasma frequency and the angular frequency of the light [32]. $N_{e}$ is the electron density, $e$ is the elementary charge, $\varepsilon_{o}$ is the permittivity of free space, and $m_{e}$ is the electron mass. Since $\omega_{p e} \ll \omega$, Eq. (8) can be linearized, and the contribution of the electrons to the index can be expressed as

$$
n_{e}-1 \approx-\frac{e^{2} \lambda^{2}}{8 \pi^{2} \varepsilon_{0} m_{e} c^{2}} N_{e}=K N_{e}
$$

where $c$ represents the speed of light. This expression illustrates the strong dependence of $n_{e}$ on the wavelength. Analogous to $\Delta n$ and $\Delta \rho$, we define $\Delta N_{e}=N_{e}-N_{e 0}$. 
Note that the expressions for $n_{H}$ and $n_{e}$ above are linear in the heavy-particle and electron densities, respectively. Thus, they also apply to the line-integrated counterparts of these refractive indices and densities.

We can then write expressions for $\Delta \bar{n}$ for each wavelength:

$$
\begin{gathered}
\Delta \bar{n}_{g}=\Delta \bar{n}_{H g}+\Delta \bar{n}_{e g}, \\
\Delta \bar{n}_{r}=\Delta \bar{n}_{H r}+\Delta \bar{n}_{e r} .
\end{gathered}
$$

The subscript $g$ refers to the 532-nm wavelength and the subscript $r$ refers to the 671-nm wavelength.

Substituting Eqs. (7) and (9) into these expressions, we can write

$$
\begin{gathered}
\Delta \bar{n}_{g}=\alpha_{g} \Delta \bar{\rho}_{H}+K_{g} \Delta \bar{N}_{e} \\
\Delta \bar{n}_{r}=\alpha_{r} \Delta \bar{\rho}_{H}+K_{r} \Delta \bar{N}_{e} .
\end{gathered}
$$

This system of two equations in two unknowns can be solved to yield

$$
\begin{gathered}
\Delta \overline{\rho_{H}}=\frac{K_{g} \Delta \overline{n_{r}}-K_{r} \Delta \overline{n_{g}}}{K_{g} \alpha_{r}-K_{r} \alpha_{g}}, \\
\Delta \overline{N_{E}}=\frac{\frac{\alpha_{g}}{\alpha_{r}} \Delta \overline{n_{r}}-\Delta \overline{n_{g}}}{\frac{\alpha_{g}}{\alpha_{r}} K_{r}-K_{g}} .
\end{gathered}
$$

To obtain the spatial distribution of $\Delta \rho_{H}$ and $\Delta N_{E}$, the line-integrated quantities are Abel inverted. Abel inversion is sensitive to noise, especially close to the axis of symmetry. The measured $\Delta \overline{\rho_{H}}$ is typically smooth enough to invert it directly using the iradon command in MATLAB. However, the system of linear equations (14) and (15) is illconditioned in $\Delta \bar{N}_{e}$ resulting in a high noise level that is further amplified if $\Delta \bar{N}_{e}$ is inverted directly. To circumvent this amplification of the noise level, we assume the following profile and fit it to the measured $\Delta \bar{N}_{e}$ data:

$$
\overline{N_{e}}(x)= \begin{cases}\frac{1}{2} \pi a N_{e 0}\left(1-\frac{x^{2}}{r_{c}^{2}}\right) & -r_{c}<x<r_{c}, \\ 0 & \text { else }\end{cases}
$$

where $r_{c}$ and $N_{e 0}$ are fit parameters. Several fit formulas (e.g., Gaussian, flat-top profile) are tested, and the above fit equation yields the best fit to the data (within the noise of the measurement). Note that $r_{c}$ can be used as a definition of the radius of the arc core, as is made clearer below.

The density $\rho_{H}$ can then be determined from $\Delta \rho_{H}$ if the density at some point in the distribution is known. We assume that outside of the arc and gas flow (at the edge of the region imaged), $\rho_{H 0}$ is known and corresponds to the density at ambient temperature $293 \mathrm{~K}$ and the measured

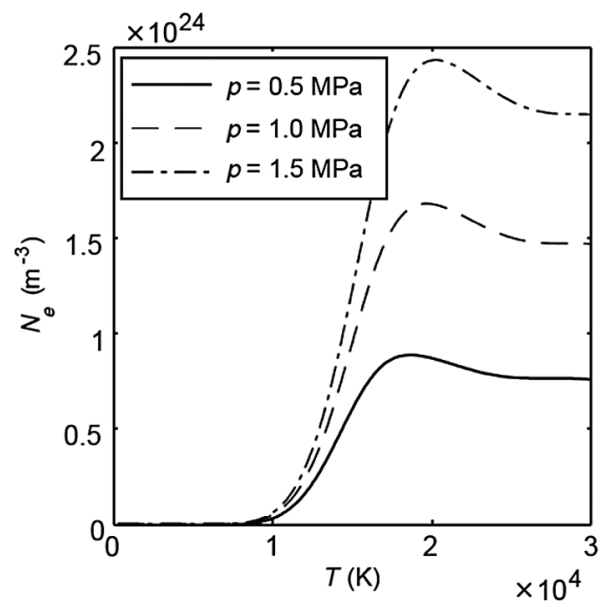

FIG. 6. $\quad N_{e}$ as a function of $T$ calculated under the assumption of LTE conditions for argon at pressures of 5, 10, and 15 bar, respectively.

stagnation point pressure. In the region outside the arc $N_{e 0}=0$ so that $\Delta N_{e}=N_{e}$.

The measured electron density can be compared to the value calculated under the assumption of local thermodynamic equilibrium. The thermodynamic equilibrium composition of argon is calculated as a function of temperature and density using the methods outlined in Refs. [33,34]. For the pressure and temperature range of interest, the most important species are $\mathrm{Ar}, \mathrm{Ar}^{+}$, and electrons. In Fig. 6, the electron density is plotted as a function of the temperature for pressures of 5, 10, and 15 bar. It can be seen that the curve peaks near $20000 \mathrm{~K}$, a temperature typical of high-current arcs [35].

\section{E. Simple axially blown arc model}

Here, we present a simplified arc model that emphasizes the main physical mechanisms that determine the size of an axially blown arc in the stagnation region of a trans-sonic flow in a nozzle system such as the one illustrated in Fig. 1. This model is based on the analysis presented by Lowke and Ludwig [14], and the arc and the physical mechanisms that influence it are illustrated in Fig. 7. It is presented here to illustrate how the pressure and the current influence the arc radius, and its results can be compared directly to the experimental measurements presented below. It is important to emphasize that only qualitative agreement can be expected with this simple, didactic model, and that the purpose of this paper is to present experimental data that can be used for validation of models and not to develop a new physical model.

It is assumed that the arc is cylindrical from the stagnation point to the nozzle throat and that the temperature $T$ inside the arc is constant. Colder gas enters the arc in the radial direction and experiences a strong acceleration in the axial direction so that it reaches the speed of sound at the nozzle throat. In a steady state, the power input from 


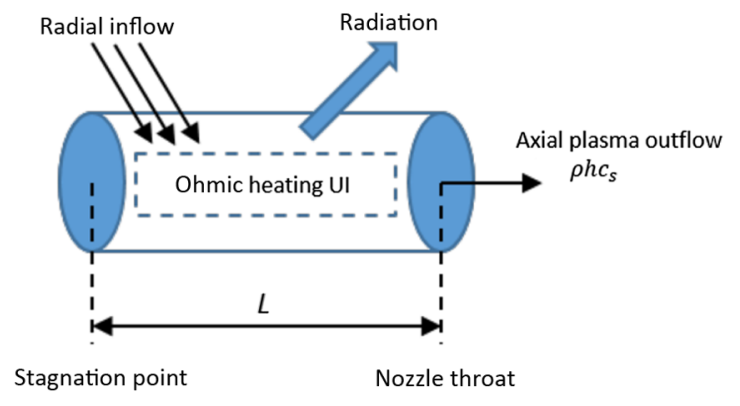

FIG. 7. Sketch illustrating the geometry of the simple model of an axially blown arc.

Ohmic heating $P_{\mathrm{el}}$ must be in equilibrium with the radiation leaving the $\operatorname{arc} P_{\text {rad }}$ and the enthalpy outflow through the nozzle $P_{\text {flow }}$ :

$$
P_{\text {el }}=P_{\text {rad }}+P_{\text {flow }} .
$$

$P_{\mathrm{el}}=U I=L I^{2} / \sigma \pi r_{c}^{2}$, where $U$ is the voltage drop across the arc segment, $I$ is the current, $r_{c}$ is the radius of the hot arc core (at temperature $T$ ), $\sigma$ is the electrical conductivity, and $L$ is the distance between the stagnation point and the nozzle throat. Furthermore, $P_{\text {flow }}=$ $\rho\left(h-h_{0}\right) c_{s} \pi r_{c}^{2}$, where $\rho$ is the plasma density, $c_{s}$ the speed of sound, $h$ the enthalpy of the hot arc plasma (at temperature $T$ ), and $h_{0}$ is the enthalpy of the inflowing ambient gas. The radiative power loss can be written as follows: $P_{\text {rad }}=\epsilon P_{\mathrm{BB}}$, where $P_{\mathrm{BB}}=\sigma_{\mathrm{rad}} 2 \pi r_{c} L T^{4}$ is the blackbody radiation leaving the plasma cylinder through its radial surface $\left(\sigma_{\text {rad }}=5.67 \times 10^{-8} \mathrm{~W} / \mathrm{m}^{2} \mathrm{~K}^{4}\right.$ is the StefanBoltzmann constant), and $\epsilon$ is the gray factor of the arc plasma. An approximation of the gray factor for an arc in $\mathrm{SF}_{6}$ is given in Ref. [36]; we use the same functional dependence but fit it to the net emission coefficient for argon (see, for example, Ref. [37]). Thus, an approximation for the gray factor is given by $\epsilon=1-\exp \left(-k p^{1.2} r_{c}^{0.5} / T\right)$ with a fit constant $k=2.7 \times 10^{-3} \mathrm{~Pa}^{-1.2} \mathrm{~m}^{-0.5} \mathrm{~K}$ and the pressure $p$. Combining the equations mentioned above yields

$$
\begin{aligned}
\frac{I^{2} L}{\sigma \pi r_{c}^{2}}= & {\left[1-\exp \left(-\frac{k p^{1.2} r_{c}^{0.5}}{T}\right)\right] \sigma_{\operatorname{rad}} 2 \pi r_{c} L } \\
& +\pi r_{c}^{2}\left(h-h_{0}\right) c_{s} \rho .
\end{aligned}
$$

For a known pressure $p$, current $I$, and a fixed temperature $T$, the quantities $\rho, h, h_{0}, c_{s}$, and $\sigma$ can be calculated for argon assuming local thermal equilibrium [33]. Thus, Eq. (18) is an implicit equation for the arc radius $r_{c}$ that can be solved numerically (for example, by using standard MATLAB routines). In the following, we compare the predictions of this simple model against the measured arc radii for various pressures and currents. A more accurate comparison to state-of-the-art computational fluid dynamics simulations is beyond the scope of this article and is planned for a subsequent publication.

\section{RESULTS}

Interferometric measurements of the electron density and heavy-particle density are carried out for several blowing pressures between 2 and 16 bar and at roughly $100 \mathrm{~A}$ for a decaying $R-C$ quasi-dc current and for a constant pressure of $11.7 \mathrm{bar}$ and currents between 50 and $200 \mathrm{~A}$. The arc and the axial gas flow are imaged in the stagnation zone of the gas flow in the test device; the region imaged is illustrated in Fig. 1.

\section{A. Processing of the interferograms}

To illustrate the processing of the interferograms, all of the steps involved in translating the measured phase shift into density and temperature profiles are presented here for a measurement made at a stagnation pressure of 10.7 bar and a current of $100 \mathrm{~A}$.

The measured phase shift (for the 532-nm laser) after unwrapping is shown in Fig. 8(a); $x=0$ represents the axis of the arc, and $y=0$ is close to the end of the hollow contact and also marks the approximate location of the stagnation point. Even without further computational analysis, the phase-shift image provides qualitative information about the size of the arc. It can be seen directly from the phase-shift image that the arc expands downstream of the stagnation point.

The line-integrated heavy-particle density and electron density can be determined at each point from the phase shifts measured at 532 and $671 \mathrm{~nm}$. To illustrate this, the measured phase shift for the 532-nm laser is plotted in Fig. 8(b) for the points along the dashed line in Fig. 8(a). The measured phase shift does not change much as a function of $y$, aside from a slight expansion of the arc, which is why we arbitrarily select the line at $y=1 \mathrm{~mm}$. Note that Fig. 8(b) shows the line-integrated change in refractive index, which is directly proportional to the phase shift $\Delta \bar{n}=\lambda \Delta \Phi /(2 \pi)$. It can be seen that in the raw phaseshift (or line-integrated refractive-index) data, the background is not flat; in other words, the phase shifts measured at the left and right edges of the image are not the same, despite the fact that the edges of the image are well outside the arc and flow region. The slope in the background is likely due to a small shift in the angle of the probe beam during the test with an arc and flow, compared to the angle during the reference shot. This small shift in the angle may result from vibration of the test device that varies from test to test. The slope and offset in the background is corrected using a simple linear fit to the data in the region outside the arc, as illustrated in Fig. 8(b); the solid line in the figure is then used for further analysis. The measured change in the line-integrated refractive index after background correction is plotted together with that for the 671-nm laser in 
(a)

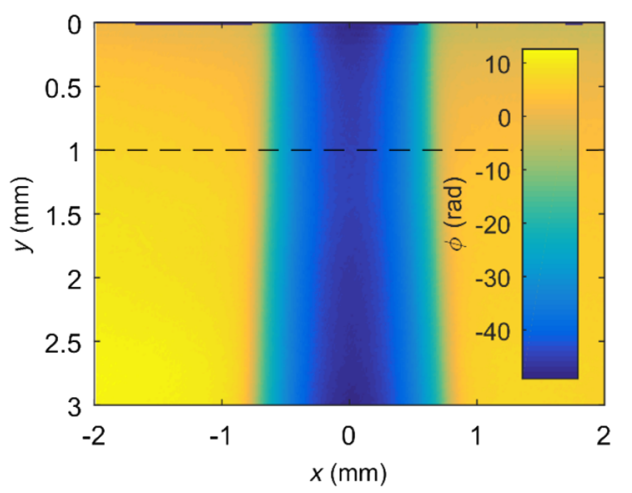

(c)

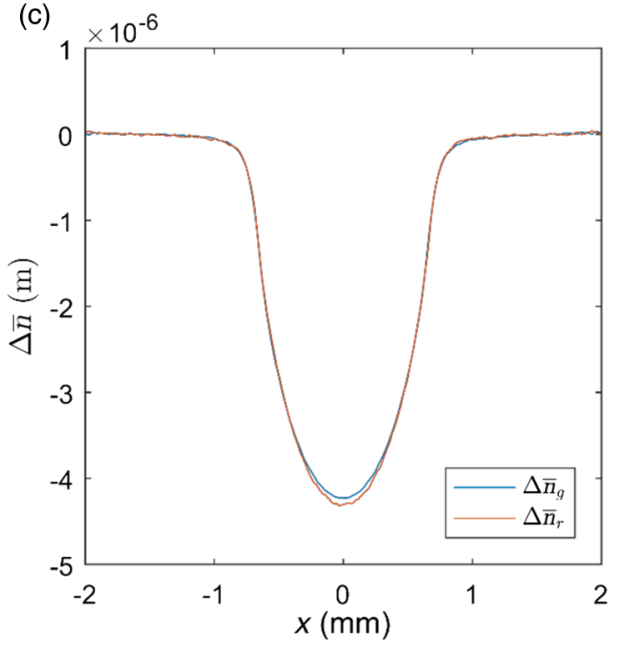

(b)

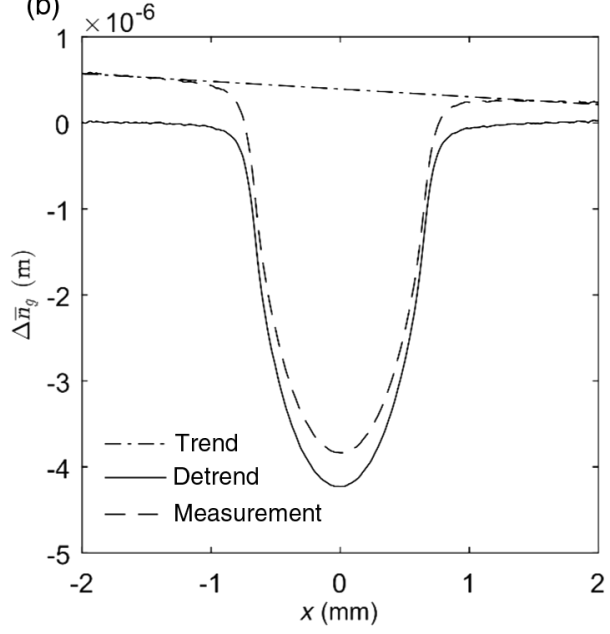

(d)

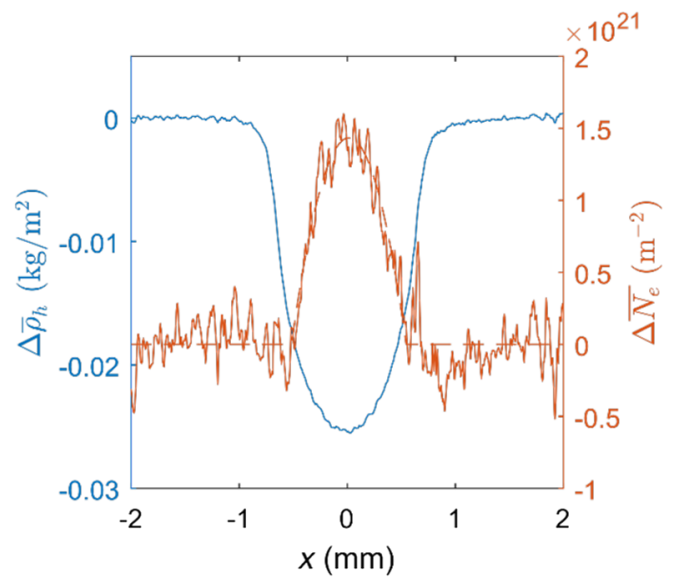

FIG. 8. (a) Measured phase shift (for the 532-nm laser) for a 100-A dc arc in argon for a stagnation point pressure of 10.7 bar. The region imaged near the stagnation point is illustrated in Fig. 1. (b) $\Delta \bar{n}_{g}$ versus $x$. The measurement is indicated by the dashed line, the linear trend by the dashed-dotted line, and the measurement after removing the slope in the background phase shift is represented by the solid line. (c) Comparison of the measured $\Delta \bar{n}_{r}$ and $\Delta \bar{n}_{g}$ after background correction. (d) Line-integrated density and $N_{e}$ for a line through the image in Fig. 8. The dashed red curve corresponds to the fit to the measured data described by Eq. (19).
Fig. 8(c); the small difference in the curves at the center of the arc is due to the presence of free electrons and their wavelength-dependent contribution to the refractive index. Using Eqs. (14) and (15), $\Delta \overline{\rho_{H}}$ and $\overline{N_{E}}$ can be determined from the results plotted in Fig. 8(c) to yield Fig. 8(d). The data plotted in Fig. 8(d) are Abel inverted to yield $\Delta \rho_{H}$ and $\Delta N_{e}$ as a function of position. Abel inversion is performed directly on the raw line-integrated heavy-particle-density $\bar{\rho}_{h}$ data (solid blue curve). Direct Abel inversion results in amplification of the noise in the electron-density measurement. However, if the smooth fitted curve is Abel inverted analytically [dashed curves in Fig. 8(d)], this noise can be eliminated without much loss in spatial resolution. Analytical Abel inversion of the fit formula given in Eq. (16) (red dashed line) is used to obtain

$$
N_{e}(r)= \begin{cases}N_{e 0} \sqrt{1-\frac{r^{2}}{r_{c}^{2}}} & -r_{c}<r<r_{c}, \\ 0 & \text { else. }\end{cases}
$$

The fit parameter $r_{c}$ in the expression for the electron density corresponds roughly to the radius of the core of the arc; it gives the radius inside of which the electron density is nonzero. The reference heavy-particle density $\rho_{H 0}=$ $17.8 \mathrm{~kg} / \mathrm{m}^{3}$ is determined using the measured stagnation point pressure of $10.7 \mathrm{bar}$ and assuming $T=293 \mathrm{~K}$. As noted above, the electron density at the reference pointwell outside the arc-is effectively zero. The resulting heavy-particle and electron densities are plotted in Fig. 9(a). The radius of the arc core $r_{c}$ is indicated by the vertical red dash-dotted line.

It can be seen that the heavy-particle density approaches zero in the center of the arc, as expected given the high temperature in the arc core. This result serves as a crosscheck of the two-color interferometry method used, since the heavy-particle density in the arc core must be of the order of $1 \%$ of $\rho_{h 0}$. The radius and the thickness of the boundary layer are quantified by fitting the measured heavy-particle density to the following model, which yields a good fit to the experimental curves:

$$
\rho(r)=\rho_{\max }+\frac{1}{2}\left(\rho_{\max }-\rho_{\min }\right)\left[\operatorname{erf}\left(\frac{r-r_{b}}{\chi}\right)-\operatorname{erf}\left(\frac{r+r_{b}}{\chi}\right)\right] .
$$



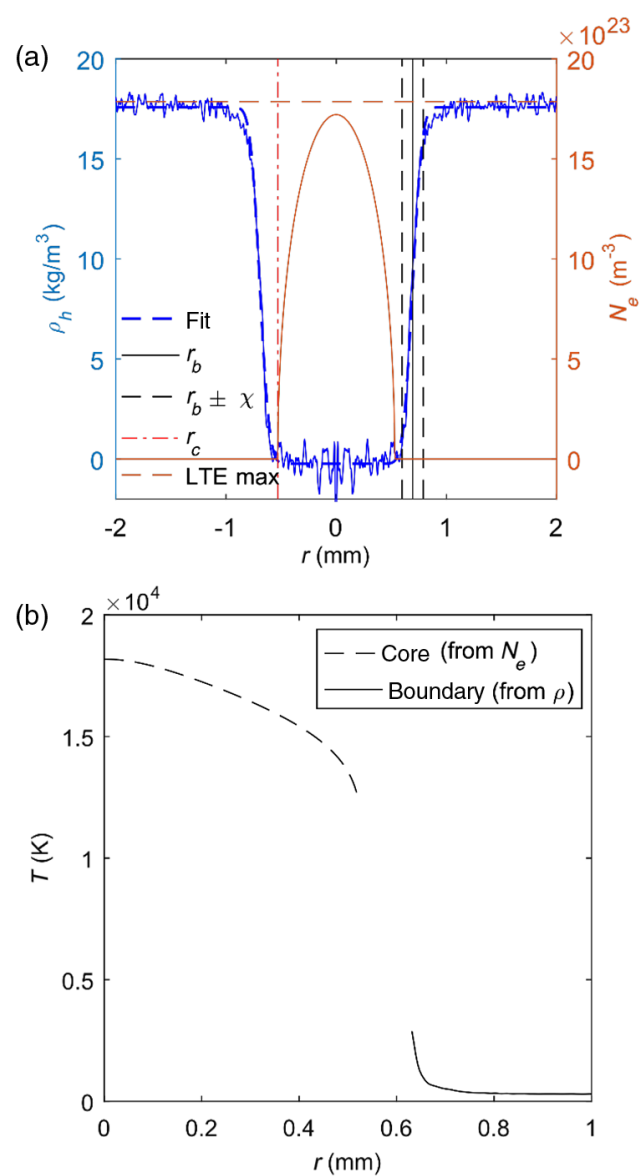

FIG. 9. (a) $\rho_{h}$ and $N_{e}$ after Abel inversion of the curves in Fig. 8(d) for a current of $100 \mathrm{~A}$ and a stagnation pressure of 10.7 bar. The radius of the boundary layer $r_{b}$ and its width $\chi$ are indicated by the solid and dashed black lines, respectively. The result obtained from Abel inversion of the fit of the line-integrated electron-density data to Eq. (19) is also shown (solid red line). The maximum electron density under LTE conditions and the radius of the hot arc core are indicated by the red dashed and dashed-dotted lines, respectively. (b) Temperature profile of the arc as derived from $\rho_{h}$ and $N_{e}$ assuming LTE conditions for $I=100 \mathrm{~A}$ and $p=10.7 \mathrm{bar}$.

In this model, $\rho_{\min }$ and $\rho_{\max }$ represent the minimum and maximum measured density, respectively. The radius of the boundary layer $r_{b}$ and its width $\chi$ are both fit parameters. The radius and width of the boundary layer are indicated by the solid and dashed black vertical curves, respectively.

Since the pressure in an axially blown arc is constant along the radial direction, the temperature profile of the arc can be determined from the measured densities (with some limitations). The temperature of the arc boundary can be obtained from the heavy-particle density by making use of the ideal-gas law

$$
T(r)=T_{0} \frac{\rho_{h 0}}{\rho_{h}(r)},
$$

which is valid up to $10000 \mathrm{~K}$ for argon. However, this relation can be used only up to temperatures of roughly $3000 \mathrm{~K}$. For higher temperatures, the value of $\rho_{h}(r)$ becomes as small as to be indistinguishable from the measurement noise. As noted in Ref. [12], this is a fundamental limitation of the index of refraction-based techniques that make use of just one wavelength. However, assuming local thermodynamic equilibrium, the temperature in the arc core can be determined from the measured electron density $N_{e}$ (a measurement that makes use of both wavelengths) and the known pressure by inverting the data plotted in Fig. 6. This inversion works best for temperatures between 12000 and $17000 \mathrm{~K}$. In this range, the electron density is very sensitive to the temperature. For lower temperatures, the electron densities are below the detection threshold of the interferometer, and for temperatures above the temperature where $N_{e}$ reaches its maximum, the electron density becomes insensitive to the arc temperature. The temperature estimate from the data plotted in Fig. 9(a) ( $p=10.7$ bar, $I=100 \mathrm{~A}$ ) is given in Fig. 9(b) (dashed line). In that case, the inversion is possible for the complete arc core, since the peak electron density at the axis was below the local thermodynamic equilibrium maximum. The temperature in the arc boundary region, which is obtained using Eq. (8), is given by the solid line and truncated at $3000 \mathrm{~K}$.

\section{B. Pressure and current variation}

The measurement and analysis illustrated for one example above is carried out for a range of stagnation pressures between roughly 2 and 16 bar, while keeping the current constant at $100 \mathrm{~A}$. The arc radius plays an important role in the cooling and ultimate interruption of an arc; the smaller the arc radius, which we define as the point where the density reaches half the ambient density $\rho_{h 0}$, the more effectively the arc can be interrupted through turbulent heat conduction and convective cooling as the current approaches zero [14]. The simple steady-state model discussed above, which balances Ohmic heating of the arc with the sum of radiative transport out of the arc and axial acceleration and removal of hot gas that radially enters the arc core, yields the dependence of the arc radius on the pressure illustrated by the red curve in Fig. 10(a) (assuming an arc core temperature of $16500 \mathrm{~K}$ ). The measurements agree well with the predicted dependence of the arc radius on the pressure, particularly if one defines the arc radius as equal to $r_{c}$, the radius of the hightemperature core of the arc. Since the model above defines the arc as a cylinder with a uniform temperature, it is expected to predict the radius of the arc core, rather than that of the arc boundary.

The peak electron density (measured at the center of the arc) increases linearly with stagnation pressure, as shown in Fig. 10(b). This result agrees well with the maximum electron density predicted by a local thermodynamic 
(a)

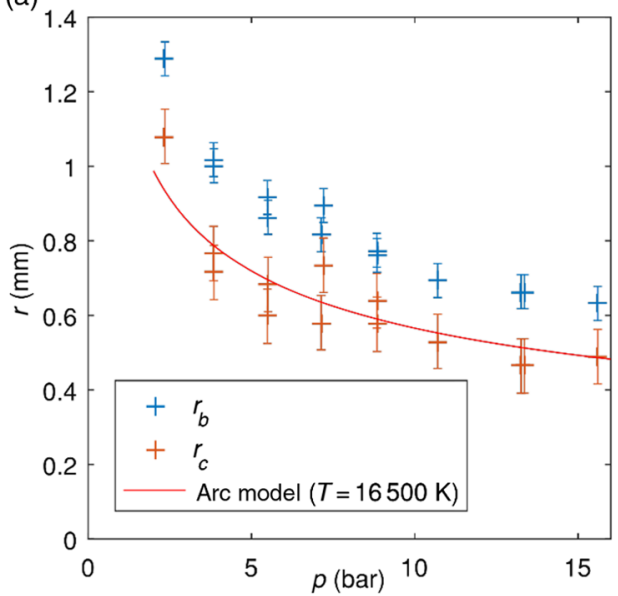

(b)

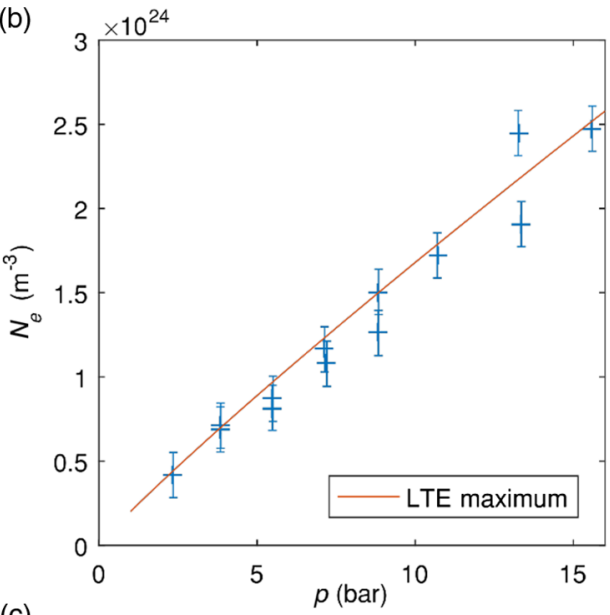

(c)

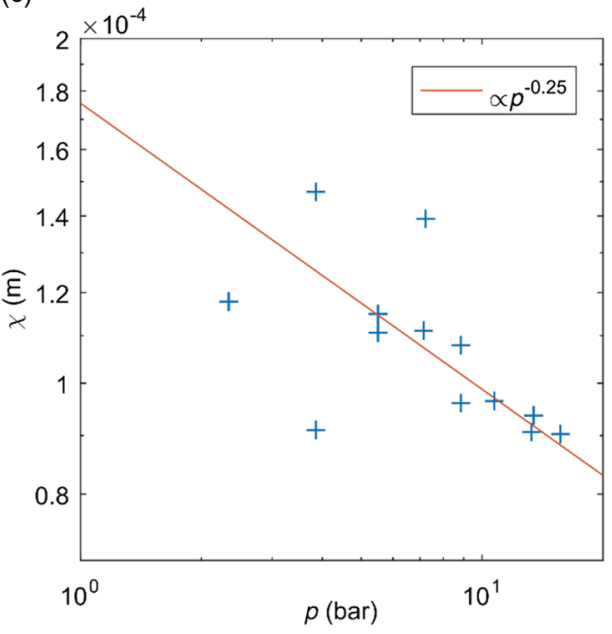

FIG. 10. (a) Measured radius of the arc as a function of stagnation point pressure for a constant current of $100 \mathrm{~A} . r_{b}$ is defined to be the radius at which $\rho_{h}=\rho_{h 0} / 2$, and $r_{c}$ the radius of the arc core is obtained from a fit to the line-integrated electron density $\overline{N_{e}}$. The red line indicates the radius as determined from the simple arc model for a temperature of $T=16500 \mathrm{~K}$. (b) Peak electron density $N_{e 0}$ as a function of stagnation point pressure for a current of $100 \mathrm{~A}$; the maximum electron density that can be reached under LTE conditions is given by the red line. (c) Arc boundary width parameter $\chi$ plotted as a function of pressure; the solid red line illustrates that $\chi$ is roughly proportional to $p^{-1 / 4}$. equilibrium model (refer to Fig. 6), which is indicated by the red curve in Fig. 10(b). The interaction between the arc and the surrounding gas flow that cools it occurs in the boundary region at the edge of the arc. As we note above, an estimate of the width of the boundary region $\chi$ is given by the dashed vertical lines in Fig. 9(a) and is determined by a fit to Eq. (20). In Fig. 10(c), the boundary width $\chi$ is plotted vs the stagnation pressure. Within the scatter of the data, this dependence can be described well by a power law (red line) demonstrating that $\chi$ is roughly proportional to $p^{-1 / 4}$.

In addition to the measurements at $100 \mathrm{~A}$, measurements are also performed for currents of approximately 50, 150, and $200 \mathrm{~A}$ (quasi-dc current measured at the point in time the interferogram is recorded). These measurements are performed for the same stagnation pressure of $11.7 \mathrm{bar}$. Figure 11(a) illustrates that if the stagnation pressure is held constant, the arc radius increases in rough agreement with the simple model presented above, which is already used to compare the measured and predicted dependence of the arc radius on pressure. This deviation may be due to an increase in the arc temperature with increasing current that is not described by the simple model.

The measured electron density in the center of the $\operatorname{arc} N_{e 0}$ depends only weakly on the current, although there is a slight trend toward an increase in $N_{e 0}$ with increasing current, as shown in Fig. 11(b). Analogous to Fig. 9(b), the temperature profile can be reconstructed from the measurement of $N_{e}$ and $\rho_{H}$. The resulting temperature profiles for each current are plotted in Fig. 11(c). They illustrate that while the arc radius increases with increasing current, the peak temperature increases only slightly. Comparing Figs. 11(b) and 10(b), it can be seen that the measured electron density $N_{e}$ is much closer to the maximum that can be reached under LTE conditions in the case of the measurements where the pressure is varied and the current is held constant than in the case where the current is varied. This difference is likely due to the fact that the nozzle geometry differs slightly between the two sets of tests due to the ablation of a small amount of nozzle material. The nozzle geometry used for the tests where the current is varied (performed earlier, with less-ablated nozzles) leads to faster acceleration from the stagnation point to the nozzle throat and, thus, may lead to slightly lower temperatures and corresponding electron densities.

The accuracy of this method, as indicated by the error bars shown in Figs. 10(a), 10(b), 11(a), and 11(b), is determined from the scatter of the measured values. For the electron density, a standard deviation of $\sigma_{n e}=1.3 \times 10^{23} \mathrm{~m}^{-3}$ is obtained after subtracting the result of a linear regression from the data plotted in Fig. 10(b). Likewise, detrending (using a smoothing spline) the measured arc radii shown in Fig. 10(a) gives a standard deviation of $\sigma_{r b}=45 \mu \mathrm{m}$ and $\sigma_{r b}=73 \mu \mathrm{m}$ for the measured radius of the arc boundary layer and the arc core, respectively. 

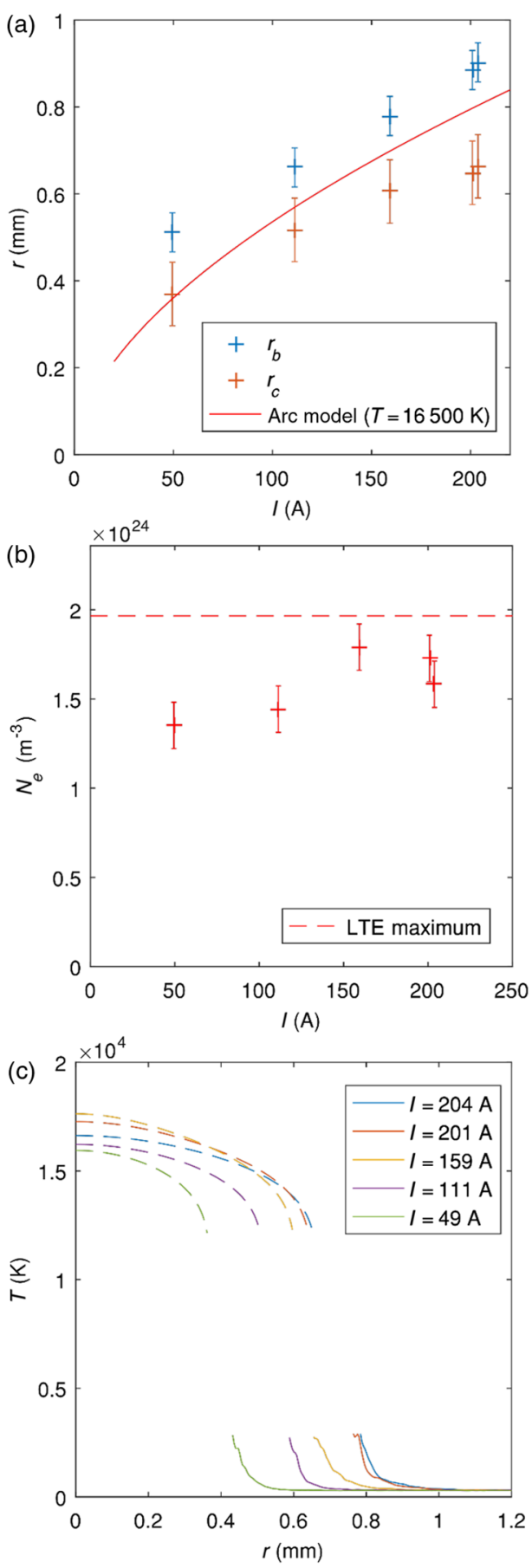

FIG. 11. (a) Measured radius of the arc as a function of current for constant pressure of 10.7 bar. $r_{b}$ is defined to be the radius at which $\rho_{h}=\rho_{h 0} / 2$, and $r_{c}$ the radius of the arc core is obtained from a fit to the line-integrated electron density $\overline{N_{e}}$. The red line indicates the radius as determined from the simple arc model for a temperature of $T=16500 \mathrm{~K}$. (b) Peak electron density $N_{e 0}$ as a function of current for a stagnation pressure of 11.7 bar; the maximum electron density that can be reached under LTE conditions is given by the red dashed line. (c) Temperature profile of the arc as derived from $\rho$ (solid lines) and $N_{e}$ (dashed lines) assuming LTE conditions for $p=11.7$ bar and currents from 50 to $200 \mathrm{~A}$. The scatter in the measured $N_{e}$ data results in an error in the temperature of roughly $\pm 500 \mathrm{~K}$ at the center of the arc.

\section{DISCUSSION}

In this work, we show that measurements of the heavyparticle and electron densities in axially blown arcs, which are the key feature of high-voltage circuit breakers and are used to switch off currents, can be made with high spatial resolution and can freeze arc dynamics on the scale of tens of nanoseconds. The measured heavy-particle density is in very good agreement with the expectation that the arc density should be effectively zero on the axis (where the temperature is very high). The measured electron density in a quasi-dc arc, in turn, agrees very well with the predictions of the maximum electron density calculated assuming local thermodynamic equilibrium [Fig. 10(b)]. For the highpressure and -temperature arc that burns in our test device, the assumption of local thermodynamic equilibrium in the arc core is well satisfied [13].

The measured arc radii for different stagnation point pressures agree quantitatively with the predictions of the simple axially blown arc model for a core temperature of approximately $16500 \mathrm{~K}$. The model assumes that radiation transported from the arc core to its boundary is used to heat up radially inflowing colder gas, which is exhausted axially. In steady state, Ohmic losses in the arc are balanced against radiative losses and convection of heat in the axial direction. This model predicts the arc radius as a function of pressure and current. Our results are in very good agreement with the predictions of the model, and the dependence on the current also agrees reasonably well with the measured data. The simple model uses a fixed arc core temperature, independent of the current, and this may explain why the radius does not increase as quickly with increasing current as predicted by the model. We choose to compare our experimental results with a simple model that highlights the main physical processes and parameters. The data presented here can be used for validation and comparison with more sophisticated models, but this is not the purpose of our work here.

Our results can be compared with a previous measurement of the electron density in an axially blown arc $[19,38]$. In that work, measurements at several radial points were performed using Thomson scattering for an arc cooled by a constant volume flow of argon of $100 \mathrm{l} / \mathrm{min}$ at a pressure of approximately 1 bar. The electron density at the center of the arc was measured to be approximately $3 \times 10^{22} \mathrm{~m}^{-3}$. Our measurements do not extend down to pressures of 1 bar (this is not feasible with our test device), but a linear extrapolation of the data to 1 bar is reasonable [refer back to Fig. 10(b)] and yields a peak electron density of $2.0 \times 10^{23} \mathrm{~m}^{-3}$. This peak electron density is roughly 7 times higher than the value reported in Refs. $[19,20]$. The measurements described in Refs. $[19,20]$ were performed in the nozzle throat, which has a diameter of $10 \mathrm{~mm}$, giving a flow speed of roughly $21 \mathrm{~m} / \mathrm{s}$. Our measurements are made in the stagnation point. The gas flow reaches the speed of sound of argon in the nozzle throat, and the associated rapid 
acceleration in opposite directions along the axis helps to stabilize the arc; the flow rate is $2200 \mathrm{l} / \mathrm{min}$ (using the speed of sound of argon at standard temperature and pressure), roughly 2 order of magnitude higher than in Refs. $[19,20]$. The optical emission images presented in Ref. [20] suggest that the arc, even in pure argon, was unstable; this may partly explain why a lower electron density was measured (below the local thermodynamic equilibrium prediction). The main factor, however, is probably the lower arc temperature of $13000 \mathrm{~K}$ calculated in Ref. [38] for the experimental conditions in Ref. [20].

Performing measurements of the electron and neutralparticle densities requires a stable, axisymmetric arc. This is achieved by designing a test device in which flow in the arc zone accelerates from rest, eliminating upstream turbulence that can destabilize the arc (even at the stagnation point of the axial flow before arc-generated turbulence can develop). Furthermore, the gas flow is supersonic in the expanding part of the nozzle. This prevents turbulence and arc instabilities that arise in the downstream section of the nozzle from propagating into the stagnation region. A stable arc permits reconstruction of the heavy-particle and electron densities for every measurement made and does not require repeated measurements (as in Ref. [12]) or selecting particularly symmetric sections of an arc [30]. It should be stressed that if the arc is unstable, it may shift from the axis in such a way that this shift is not evident in a single projected image. In this case, the assumption of symmetry can lead to incorrect results when axisymmetry is assumed to reconstruct the index of refraction distribution from the line-integrated measurements $[12,30]$.

A stable, axisymmetric arc has the further advantage that spatial averaging is not required to smooth small asymmetries. Combined with spatial carrier wave interferometry, this lack of a need to perform a spatial average results in a high resolution. The spatial resolution is not defined by the measurement technique alone but also depends strongly on the magnification (which can vary greatly between different implementations of a technique). However, the available pixel resolution can be used to make a rough comparison of the different techniques. In the case of spatial carrier wave interferometry, the initial resolution of the camera of 16 megapixels is reduced to roughly 1 megapixel by selecting a small region in Fourier space. In the case of speckle imaging [12], shifts in the speckle pattern can be detected only by comparing regions of pixels. The resolution of the camera chip is reduced by the size of these regions-for example, from 16 megapixels to 62.5 kilopixels if $16 \times 16$ pixel regions are used. A Shack-Hartmann interferometer functions by detecting the angular offset of the light rays passing through an array of microlenses; to do so, multiple pixels must be assigned to a single microlens. This assignment of multiple pixels to a single microlens reduces the spatial resolution, for example, in the case of the setup described in Ref. [11], from more than 1 megapixel to roughly $30 \times 30$ pixels (microlens focal spots). The high resolution achieved with spatial carrier wave interferometry (combined with the stability of the arc in our test device) permits straightforward identification of the arc boundary and even allows the width of this boundary region to be quantified [refer back to Figs. 9(a) and 10(a)].

The use of an interferometric technique with an external light source is advantageous in that it can be used to investigate the arc at or even after current zero, when there is insufficient light emission to perform emission spectroscopy. The use of two lasers with different wavelengths permits the heavy-particle density and the electron density to be determined simultaneously by solving a system of two equations in two unknowns. The wavelengths we use, which are restricted by the pulsed lasers available to us, are not optimal for measuring the electron density. In fact, by selecting two lasers with a wavelength of 355 and $671 \mathrm{~nm}$ (or even 355 and $1064 \mathrm{~nm}$ ), the sensitivity (signal-to-noise ratio) of the technique can be doubled (or even increased sixfold). This might allow direct Abel inversion of $\bar{N}_{e}$ and, consequently, a more accurate determination of the radial distribution of the electron density. In particular, this increase in the sensitivity is beneficial for measurements after current zero, where the electron density decays rapidly. State-of-the-art measurements in the recovery phase are based on Thomson scattering and have limited spatial resolution [20]. A well-resolved density profile might allow an even more detailed comparison between experiment and recent models of the recovery phase.

The method for determining the electron density is not limited to argon; argon is selected for these first measurements because the ratio of the Gladstone-Dale coefficients $\alpha_{g} / \alpha_{r}$ at the two laser wavelengths [see Eq. (15)] is almost constant up to a temperature of roughly $10000 \mathrm{~K}$. In other gases, such as $\mathrm{CO}_{2}$, this ratio depends weakly on the temperature and can lead to a small systematic error. However, if the difference in wavelengths is large enough, this small effect can be neglected, for example, in $\mathrm{CO}_{2}$ and air [39]. The approach presented here can, thus, readily be extended to other gases relevant for circuit breakers. The use of pulsed lasers leads to an integration time of several ten nanoseconds so that transient phenomena can be studied as well (for example, the interaction of the gas flow with the arc at current zero).

\section{ACKNOWLEDGMENTS}

We thank Sandor Simon, Philipp Jabs, René Salzmann, and Britta Schiedlauske for their assistance in setting up and carrying out the experiments. We thank Martin Seeger for valuable input and useful discussions, especially on the topic of the simple arc model. We thank Marcelo Buffoni for his help, in particular, with regard to the design of the nozzle. We thank Frank Kassubek for calculating the gray factor used in the simple arc model. 
[1] B. Franklin, A letter of Benjamin Franklin, Esq; to Mr. Peter Collinson, FRS concerning an electrical kite, Phil. Trans. R. Soc. London 47, 565 (1751).

[2] A. Iordanidis and C. Franck, Self-consistent radiation-based simulation of electric arcs: II. Application to gas circuit breakers, J. Phys. D 41, 135206 (2008).

[3] H. Nordborg and A. A. Iordanidis, Self-consistent radiation based modelling of electric arcs: I. Efficient radiation approximations, J. Phys. D 41, 135205 (2008).

[4] L. Fagiano and R. Gati, On the order reduction of the radiative heat transfer model for the simulation of plasma arcs in switchgear devices, J. Quant. Spectrosc. Radiat. Transfer 169, 58 (2016).

[5] V. Aubrecht and J.J. Lowke, Calculations of radiation transfer in $\mathrm{SF}_{6}$ plasmas using the method of partial characteristics, J. Phys. D 27, 2066 (1994).

[6] F. Reichert, J.-J. Gonzalez, and P. Freton, Modelling and simulation of radiative energy transfer in high-voltage circuit breakers, J. Phys. D 45, 375201 (2012).

[7] W. Hermann, U. Kogelschatz, L. Niemeyer, K. Ragaller, and E. Schade, Investigation on the physical phenomena around current zero in HV gas blast breakers, IEEE Trans. Power App. Syst. 95, 1165 (1976).

[8] W. Hermann, U. Kogelschatz, L. Niemeyer, K. Ragaller, and E. Schade, Experimental and theoretical study of a stationary high-current arc in a supersonic nozzle flow, J. Phys. D 7, 1703 (1974).

[9] U. Kogelschatz, Application of a simple differential interferometer to high current arc discharges, Appl. Opt. 13, 1749 (1974).

[10] U. Kogelschatz and W. R. Schneider, Quantitative schlieren techniques applied to high current arc investigations, Appl. Opt. 11, 1822 (1972).

[11] Y. Inada, S. Matsuoka, A. Kumada, H. Ikeda, and K. Hidaka, Multi-time electron density imaging over arc discharges around the current zero point, J. Phys. D 47, 175201 (2014).

[12] P. Stoller, E. Panousis, J. Carstensen, C. B. Doiron, and R. Färber, Speckle measurements of density and temperature profiles in a model gas circuit breaker, J. Phys. D 48, 015501 (2015).

[13] A. Gleizes, Perspectives on thermal plasma modelling, Plasma Chem. Plasma Process. 35, 455 (2015).

[14] J. J. Lowke and H. C. Ludwig, A simple model for highcurrent arcs stabilized by forced convection, J. Appl. Phys. 46, 3352 (1975).

[15] E. Panousis, M. Bujotzek, and T. Christen, Arc cooling mechanisms in a model circuit breaker, IEEE Trans. Power Delivery 29, 1806 (2014).

[16] D. Eichhoff, A. Kurz, R. Kozakov, G. Gött, D. Uhrlandt, and A. Schnettler, Study of an ablation-dominated arc in a model circuit breaker, J. Phys. D 45, 305204 (2012).

[17] R. Kozakov, M. Kettlitz, K.-D. Weltmann, A. Steffens, and C. M. Franck, Temperature profiles of an ablation controlled arc in PTFE: I. Spectroscopic measurements, J. Phys. D 40, 2499 (2007).

[18] S. Vacquie, A. Gleizes, and H. Kafrouni, Measurements of electron density in a $\mathrm{SF}_{6}$ arc plasma, J. Phys. D 18, 2193 (1985).
[19] K. Tomita, D. Gojima, K. Nagai, K. Uchino, R. Kamimae, Y. Tanaka, K. Suzuki, T. Iijima, T. Uchii, and T. Shinkai, Thomson scattering diagnostics of decay processes of $\mathrm{Ar} / \mathrm{SF}_{6}$ gas-blast arcs confined by a nozzle, J. Phys. D 46, 382001 (2013).

[20] K. Tomita, D. Gojima, T. Shimizu, K. Uchino, T. Nakano, Y. Tanaka, K. Suzuki, T. Iijima, and T. Shinkai, Thomson scattering diagnostics of $\mathrm{SF}_{6}$ gas-blasted arcs confined by a nozzle under free-recovery conditions, J. Phys. D 48, 265201 (2015).

[21] M. Stoffels, S. Simon, P. G. Nikolic, P. Stoller, and J. Carstensen, Development of a multiperspective optical measuring system for investigating decaying switching arcs at the nozzle exit of circuit breakers, Appl. Opt. 56, 2007 (2017).

[22] W. Bötticher, U. Kogelschatz, and E. Schade, Untersuchung quasistationärer Lichtbögen hoher Leistung bei starker axialer Gasströmung, Z. Naturforsch. 27A, 1433 (1972).

[23] K. A. Goldberg and J. Bokor, Fourier-transform method of phase-shift determination, Appl. Opt. 40, 2886 (2001).

[24] M. Takeda, H. Ina, and S. Kobayashi, Fourier-transform method of fringe-pattern analysis for computer-based topography and interferometry, J. Opt. Soc. Am. 72, 156 (1982).

[25] K. Muraoka, M. Hamamoto, and M. Akazaki, Studies of an impulse spark using two-wavelength interferometry, Jpn. J. Appl. Phys. 19, L293 (1980).

[26] M. J. Hargather and G. S. Settles, A comparison of three quantitative schlieren techniques, Opt. Lasers Eng. 50, 8 (2012).

[27] D. C. Ghiglia and M. D. Pritt, Two-Dimensional Phase Unwrapping: Theory, Algorithms, and Software (Wiley, New York, 1998), Vol. 4.

[28] C. Smith and B. Spottiswoode, GoldsteinUnwrap2D_r1, http://ch.mathworks.com/matlabcentral/fileexchange/29497goldsteinunwrap2d-r1, 2016.

[29] G. S. Settles, Schlieren and Shadowgraph Techniques: Visualizing Phenomena in Transparent Media (Springer, New York, 2001).

[30] Y. Inada, S. Matsuoka, A. Kumada, H. Ikeda, and K. Hidaka, Shack-Hartmann type laser wavefront sensor for measuring two-dimensional electron density distribution over extinguishing arc discharge, J. Phys. D 45, 435202 (2012).

[31] W. Burton, The refractive index and dispersion of light in argon and helium, Proc. R. Soc. A 80, 390 (1908).

[32] A. Piel, Plasma Physics: An Introduction to Laboratory, Space, and Fusion Plasmas (Springer Science \& Business Media, New York, 2010).

[33] C. Doiron and K. Hencken, Calculation of thermodynamic and transport properties of thermal plasmas based on the Cantera software toolkit, in Proceedings of ESCAMPIG XXII (Greifswald, Germany, 2014), http://www .escampig2014.org/downloads/xxii_escampig_abstract_ booklet_final.pdf.

[34] W. R. Smith and R.W. Missen, Chemical Reaction Equilibrium Analysis: Theory and Algorithms (Wiley, New York, 1982).

[35] A. Gleizes, J. J. Gonzalez, and P. Freton, Thermal plasma modelling, J. Phys. D 38, R153 (2005). 
[36] M. Seeger, L. Niemeyer, T. Christen, M. Schwinne, and R. Dommerque, An integral arc model for ablation controlled arcs based on CFD simulations, J. Phys. D 39, 2180 (2006).

[37] M. Wendt, Net emission coefficients of argon iron plasmas with electron Stark widths scaled to experiments, J. Phys. D 44, 125201 (2011).

[38] K. Murai, T. Nakano, Y. Tanaka, Y. Uesugi, T. Ishijima, K. Tomita, K. Suzuki, T. Iijima, and T. Shinkai, The LTE thermofluid simulation of $\mathrm{Ar} / \mathrm{SF}_{6}$ gas-blast arcs in a nozzle space in an arc device, IEEE Trans. Power Energy 136, 741 (2016).

[39] Y. Inada, A. Kumada, H. Ikeda, K. Hidaka, T. Nakano, K. Murai, Y. Tanaka, and T. Shinkai, Comparative study on extinction process of gas-blasted air and $\mathrm{CO}_{2}$ arc discharge using two-dimensional electron density imaging sensor, J. Phys. D 50, 175202 (2017). 